\title{
High resolution melting curve analysis with MATLAB-based
}

\section{program}

\author{
Huaizhong $\mathrm{Li}^{1 *}$, Ruiting $\mathrm{Lan}^{2}$, Niancai Peng ${ }^{3}$, Jing Sun ${ }^{4}$, Yong $\mathrm{Zhu}^{1}$ \\ ${ }^{1}$ Griffith School of Engineering, Gold Coast campus, Griffith University, QLD 4222, \\ Australia \\ ${ }^{2}$ School of Biotechnology and Biomolecular Sciences, The University of New South \\ Wales, Sydney, NSW 2052, Australia \\ ${ }^{3}$ School of Mechanical Engineering, Xi'an Jiaotong University, Xi'an, China \\ ${ }^{4}$ Menzies Health Institute Queensland and School of Medicine, Griffith University, \\ QLD 4222, Australia
}

* Corresponding Author: Dr Huaizhong Li, Griffith School of Engineering, Gold Coast campus, Griffith University, QLD 4222, Australia. E-mail: lihuaizhong@gmail.com; h.li@griffith.edu.au. Telephone: +61 (7) 5552 8252; Facsimile: +61 (7) 55528062

Abstract. High resolution melting curve analysis (HRM) is an emerging new method for interrogating and characterizing DNA samples. It has been used as a powerful tool for gene mutation and single-nucleotide polymorphism (SNP) detection with high throughput and low cost. Commercially available HRM analysis systems are mostly proprietary and expensive. It lacks the flexibility for the end users and researchers to 
incorporate new analysis algorithms into the existing system. This paper presents the development of a MATLAB-based open software program for high resolution melting curve analysis. Key analysis functions, such as to obtain the first derivative curve using Savitzky-Golay filter, to identify the melt region, subtraction of background fluorescence and curve normalization, are introduced, followed by case studies of HRM analysis using the developed software program.

Keywords: High resolution melting curve analysis (HRM); derivative curve; SavitzkyGolay filter; exponential background subtraction; data linearization; MATLAB

\section{Introduction}

High resolution melting curve analysis (HRM) is a relatively new technique for fast, high-throughput post-PCR analysis of genetic mutations or variance in nucleic acid sequences. It enables researchers to rapidly detect and categorize genetic mutations, to identify new genetic variants without sequencing (gene scanning) or to determine the genetic variation in a population prior to sequencing [1].

HRM technology was made possible by dsDNA dyes that detect heteroduplexes, dedicated high-resolution melting instruments, and specific software for data analysis. In this method, real-time PCR instrumentation is used for the amplification of the region of interest in the presence of a specialized dsDNA binding dye [2][3][4]. This dye is highly fluorescent when bound to dsDNA and poorly fluorescent in the unbound state. This change allows the user to monitor the DNA amplification during 
PCR. The amplified target is then gradually denatured by increasing the temperature in small increments. The amplified target denatures gradually, releasing the dye, which results in a drop in fluorescence. By monitoring the fluorescence continuously and plotting it against the temperature, a characteristic melting profile can be produced. The melting profile depends on the length, GC content, sequence and heterozygosity of the amplified target. When set up correctly, HRM is sensitive enough to allow the detection of a single base change between otherwise identical nucleotide sequences [5][6]. The highest rate of fluorescence decrease is generally at the melting temperature (Tm) of the DNA sample. The $T_{m}$ is defined as the temperature at which $50 \%$ of the DNA sample is double-stranded and $50 \%$ is singlestranded. The $T_{m}$ is typically higher for DNA fragments that are longer and/or have a high GC content. The temperature increases for HRM are typically in $0.008-0.2{ }^{\circ} \mathrm{C}$ steps. This allows a much more detailed analysis of the melting behaviour. HRM sensitivity and reliability has been improved with the use of a variety of new dsDNA intercalating dyes [6].

The HRM data is analysed by specific software and the results are displayed to help discriminate between sequence variants. For a given set of HRM data, the absolute sample fluorescence depends on the sample volume, instrument optics, and PCR amplification. Currently the widely used HRM data processing approaches include normalization, overlay, derivative and difference plots, etc. [6]. Methods for modelling and analysis of HRM assays have been presented by Palais and Wittwer [7]. These include filtering the melting signal from raw data based on a model of fluorescent background, predicting the melting curves of pure duplexes and their mixtures using thermodynamic parameters, etc. Commercial HRM analysis products 
and software packages from different manufacturers have been on the market for nearly one decade. However, these analysis systems are mostly proprietary. Due to the complexity of the DNA melting process and possible variations in the real time PCR assays, there exist some intrinsic limitations using earlier computational approaches to model and analyse this complex process [2][7]. It is noted that some novel additions to the general standard approach have yet to be implemented due to the proprietary features of the commercial system.

Development of innovative approaches is needed, and also an open software platform for researchers to implement new algorithms. An example is DivMelt which is an R package HRM Diversity Assay Analysis Tool designed for researchers to calculate genetic diversity measures from DNA melting curve data [8]. Considering the fact that MATLAB is one of the most widely used software tools for researchers and engineers dealing with signal processing, the development of an open platform HRM curve analysis software package based on MATLAB would be meaningful to the researchers and developers, which is to be presented in this paper. It is believed that such a package will enhance the throughput and objectivity of measurements generated with the HRM analysis.

\section{Methodology}

For HRM curve analysis, there are three major tasks in most commercial software [9], which include (i) Normalization and background subtraction; (ii) Melting temperature identification; and (iii) Difference curve construction. These data processing 
procedures allow better discrimination between melt curves of different genotypes. After obtaining the raw melt curve plot, a negative derivative curve is constructed to identify the melting temperature $T_{m}$, and a difference curve is derived by subtracting a known reference curve from the fluorescence curve.

The implementation of the HRM curve analysis is by using MATLAB procedural programming scripts. A flowchart of the processing procedure is given in Fig. 1.

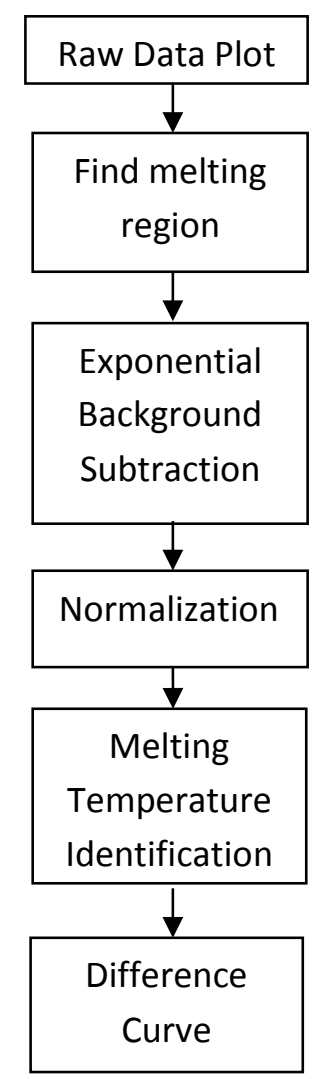

Fig. 1. Flowchart of the HRM data processing procedure

\section{$\underline{\text { Melt Region Identification }}$}

An amplicon melt region is the temperature range in which the amplicons denature. It is between the melt start temperature $T_{1}$, and melt end temperature $T_{2}$. The amplicon 
melt region is to be identified for two purposes, i.e., to enable exponential background subtraction and to get a novel feature called HRM score. The HRM score is defined as the width of the melting region and is an assay which allows rapid quantification of DNA diversity, a possible feature of interest in some studies [8].

The method used in finding the melting region is based on the negative first derivative curve and the angle formed by the curve. It is widely known that digital differentiation results in amplification of noise. Of such, using smoothing and filtering functions can be applied in obtaining the first derivative curve. The use of SavitzkyGolay smoothing filter to obtain the first derivative curve in HRM curve analysis has been recommended by Palais and Wittwer [10].

The Savitzky-Golay filter is a FIR smoothing filter which is also known as polynomial smoothing, or least-squares smoothing filter. Savitzky-Golay filter works by differentiation of a moving window of least-squares polynomial fit using a table of convolution coefficients [11]. The Savitzky-Golay filter can also be used as a differentiation algorithm with the appropriate choice of input arguments. It is thus capable to combine differentiation and smoothing into one algorithm. The data smoothing aspect in Savitzky-Golay method should be done with care lest features are lost. For example, multiple nearby melting peaks characteristic in heterozygous samples can be difficult to resolve if excessive smoothing due to choosing excessively large fitting windows or inadequate polynomial degree. A polynomial degree of two and a window length encompassing $1{ }^{\circ} \mathrm{C}$ has been used by Wittwer et al. [1]. Another 
smoothing option for HRMA implemented by Cousins et al. [8] with DivMelt is the Locally Weighted Least Squares Scatterplot Plotting, also known as LOWESS.

The negative first derivative curve in this study is obtained using the Savitzky-Golay filter, utilising the MATLAB Savitzky-Golay filter design function sgolay( ). The function $[b, g]=\operatorname{sgolay}(k, f)$ designs a Savitzky-Golay FIR smoothing filter $b$ and also the matrix $g$ of differentiation filters. The polynomial order $k$ is set as 2 , and the frame size, $f$, is set as $f=\operatorname{RecWindow} * 2+1$, where RecWindow is the number of samples within $1{ }^{\circ} \mathrm{C}$. Using an array of the measured raw fluorescence melting curve data, $F($ ), the $n$-th data point at the negative first derivative curve is found by apply the MATLAB $\operatorname{dot}()$ function to return the scalar product of the two vector segments, which is

$\operatorname{DerivFluo(n)}=-\operatorname{dot}(g(:, 2), F(n-\operatorname{RecWindow}: n+\operatorname{RecWindow}))$;

The output of the smoothed negative first derivative curve is used as input for the HRMStart.m and HRMEnd.m functions to find the temperature where the melting starts and ends respectively. The melting start and end temperatures are found by finding points in the negative derivative curve where the angle formed between the tangent to the points and the baseline satisfies a user specified angle, which is generally set as $30^{\circ}, 40^{\circ}$ or $50^{\circ}$. HRMStart.m and HRMEnd.m implements the method by fitting a straight line through a user specified window of points on the negative first derivative curve. The curve fitting is performed with least squares, utilising the MATLAB polyfit( ) function. The region angle is found by using the inverse tangent to the slope of the fit in relation to the $\mathrm{x}$-axis with the MATLAB inverse tangent function, atand( ). The window of points is moved until a region curve angle which is 
equal to or surpasses the user specified angles $\theta_{1}$ for melting start and $\theta_{2}$ for melting end is encountered. The temperature at the region where this occurs is then identified as the melting start or melting end respectively. In the current implementation, user specified angle $\theta_{1}$ of $50^{\circ}$ for start of melting region and $\theta_{2}$ of $30^{\circ}$ for end of melting region was chosen. These angles are chosen as recommended in [8]. The difference in $\theta_{1}$ and $\theta_{2}$ can be attributed to the fact that there are steeper slopes in higher temperature sides and more variable slope in the lower temperature sides.

\section{Exponential Background Subtraction (EBS) and Normalization}

The melting curve requires subtraction of background fluorescence and normalization for better accuracy and easier comparison of melting transition. Variation in fluorescence in a melting curve does not only occur due to temperature response. Factors that affect the relationship include fluorescence from non-target DNA, thermal variation in response of dye, non-linear response to hybridization and fluorescence behaviour at changing temperatures [7].

In order to extract the melting curve independent of the background fluorescence, background subtraction is performed [7]. There are currently two major methods for background subtraction. The first method, referred to as the baseline method, has been widely used in many studies [12]. The method extracts the melt curve from the raw data by the following approximation:

$$
M(T)=\frac{F(T)-L_{0}(T)}{L_{1}(T)-L_{0}(T)}
$$


where $M(T)$ is the approximated melt curve from the raw melting curve $F(T) . L_{0}(T)$ and $L_{1}(T)$ are given as straight line fits in temperature ranges above and below the melting transition.

Another background subtraction method is the Exponential Background Subtraction or EBS [7], which offers a more robust solution that accommodates for cases of multiple small amplicons and unlabeled probes where the baseline method fails. EBS when coupled with normalization enhances accuracy by eliminating background signals which might interfere with the analysis of data and makes comparison of the melt curves easier. Unfortunately, EBS has not been incorporated into any commercial software so far. In EBS, the raw fluorescence $F(T)$ is modelled as composing of the melting curve $M(T)$ and an exponentially decaying background $B(T)$. It can be expressed using the following mathematical model [10]:

$F(T)=M(T)+B(T)$

The modelling of $B(T)$ as an exponential function follows the widely known relation between fluorescence with increasing temperature.

The first step of EBS process is the determination of temperatures $T_{L}$ and $T_{R}$ which are the points no melting occurs. $T_{L}$ and $T_{R}$ are sufficiently below and above the melting temperature $T_{m}$ such that the derivatives of the melting curve component $\mathrm{M}(\mathrm{T})$ at these temperatures are effectively zero. Essentially, the temperatures should correspond to the equations given in Equations 3 and 4 below. 
$\frac{d M}{d T}(\mathrm{TL})=\frac{d M}{d T}(\mathrm{TR})=0$

$F^{\prime}(\mathrm{TL})=B^{\prime}(\mathrm{TL})$ and $F^{\prime}(\mathrm{TR})=B^{\prime}(\mathrm{TR})$

The background noise model $B(T)$ is approximated as an exponential function which can be expressed as

$B(T)=B 0+C e^{a(T-\mathrm{TL})}$

A number of options are available in the identification of $T_{L}$ and $T_{R}$ such as; measurement in generating raw melting curve, user specification of the regions and an automatic positioning algorithm [10]. In this work, the choice of $T_{L}$ and $T_{R}$ can be specified by the user with the help of the results in the previous procedure. The user choice can be facilitated by the resultant amplicon melting regions. Reasonable points outside the determined amplicon melting region are chosen and the data to be processed by the algorithm is truncated to fit the range of $T_{L}$ and $T_{R}$.

Following the choice of $T_{L}$ and $T_{R}$, the parameters $a$ and $C$ used in EBS can be found by substituting the relevant information:

$$
\begin{aligned}
& a=\frac{\ln \frac{d F}{d T}\left(T_{R}\right)-\ln \frac{d F}{d T}\left(T_{L}\right)}{T_{R}-T_{L}} \\
& C=\frac{\frac{d F}{d T}\left(T_{L}\right)}{a}
\end{aligned}
$$

Thus it allows the removal of the background from the original melt curve with the following equation: 
Finding parameter $a$ in Equation 6 requires the acquisition of the values of $\frac{d F}{d T}\left(T_{R}\right)$ and $\frac{d F}{d T}\left(T_{L}\right)$, i.e., the slopes of the curve at $T_{L}$ and $T_{R}$. The slopes are determined by fitting an exponential function of the form $y=k e^{\beta x}$ to the temperatures $T_{L}$ and $T_{R}$ and points encompassing $1^{\circ} \mathrm{C}$ after it and differentiating. This fitting can be performed by using a curve fitting method known as data linearization [13]. The process of data linearization method is as follows [13].

A logarithm is taken for both sides of the $y=k e^{\beta x}$ exponential form to give

$\ln (y)=\beta x+\ln (k)$

In the context of this implementation, $y$ is the fluorescence data and $x$ is the temperature data within the $1^{\circ} \mathrm{C}$ window. A few new variables are introduced to obtain a linear relationship

$\mathrm{Y}=\ln (y), X=x$ and $B=\ln (k)$

$\mathrm{Y}=\beta X+B$

The original data variables $\mathrm{x}, \mathrm{y}$ in the $\mathrm{x}-\mathrm{y}$ plane are transformed into points $X_{k}, Y_{k}=x_{k}, \ln \left(y_{k}\right)$ in the new $\mathrm{X}-\mathrm{Y}$ plane. The last squares line of $\mathrm{Y}=\beta \mathrm{X}+\mathrm{B}$ is fit into the $X_{k}, Y_{k}$ points. Normal equations for finding $\beta$ and $\mathrm{B}$ are:

$$
\begin{aligned}
& \left(\sum_{\mathrm{k}=1}^{\mathrm{n}} \mathrm{X}_{\mathrm{k}}^{2}\right) \beta+\left(\sum_{\mathrm{k}=1}^{\mathrm{n}} \mathrm{X}_{\mathrm{k}}^{2}\right) \mathrm{B}=\sum_{\mathrm{k}=1}^{\mathrm{n}} \mathrm{X}_{\mathrm{k}} \mathrm{Y}_{\mathrm{k}} \\
& \left(\sum_{\mathrm{k}=1}^{\mathrm{n}} \mathrm{X}_{\mathrm{k}}\right) \beta+\mathrm{NB}=\sum_{\mathrm{k}=1}^{\mathrm{n}} \mathrm{Y}_{\mathrm{k}}
\end{aligned}
$$


Parameter $k$ of $y=k e^{\beta x}$ is computed by $k=e^{B}$.

The curve fitting results in the exponential equation, which is then differentiated to get $\frac{d F}{d T}\left(T_{R}\right)$ and $\frac{d F}{d T}\left(T_{R}\right)$ required in Equation 6. Substituting relevant values gets parameter $a$ and $C$ from Equations 6 and 7. Obtaining the parameter, $a$ and $C$ allowed the removal of the background from the original melt curve by substituting the relevant values as expressed in Equation 8.

Following exponential background subtraction, the melt fluorescence curve should exhibit horizontal slope before and after the melting transition. Normalization of the fluorescence level can be performed to better compare the melting transition. A widely applied method of normalization is scaling the curve to a range of 0 and 100 with a linear shift by applying a linear shift. In this implementation, the maximum fluorescence is scaled to 100 while the minimum fluorescence value is scaled to 0 . The linear shift is performed by applying this algorithm:

$\operatorname{Normal} M(T)=\frac{100(M(T)-\min M(T))}{(\operatorname{Max} M(T)-\min M(T))}$

\section{Melting Temperature $\left(\mathrm{T}_{\mathrm{m}}\right)$ Identification}

The melting temperature $T_{m}$, defined as the point where $50 \%$ of the dsDNA has denatured, is a feature of interest in many HRM analyses in discriminating genetic variants. Finding the $T_{m}$ is essential for analysis of homozygote variants where only a shift in $T_{m}$ signifies a variant genotype. The $T_{m}$ is determined as the peak of the negative derivative curve. First, the Savitzky-Golay filter function is used to derive 
the first derivative curves and subsequently the values are changed into negative. Next, a MATLAB function $\max ()$ is used to find the location of the highest values in each curve which determined $T_{m}$, the melting temperature.

\section{Difference curve}

The preferred analytical component for HRM analysis is the use of difference curve, where the difference between all fluorescence curves and a reference curve is plotted. Obtaining the difference curve is often the preferred method in distinguishing different genotype melt curves. The plot makes distinguishing clusters of genotypes easier and more accurate by amplifying differences in melt curve shapes. The reference curve also referred to as the baseline is usually a known wild type curve or the average of all wild type curves to facilitate clusters around the horizontal axis. However the use of a composite median reference curve has also been found in a platform specific workflow such as performed in [14] and [15].

The current implementation can derive two sorts of reference curves, i.e., the known genotype reference curve and the composite median curve. For the known genotype reference curve, the difference plots are obtained by systematically subtracting all melt curves with a labelled curve. For the median reference curve, the median reference curve is obtained by finding the median fluorescence among all the data sets for each temperature point in the melt curve using the MATLAB median function. Each melt curve in the data set is then subtracted with the median curve. In the current study, the composite median is used. 


\section{Results}

Two sets of raw HRM data acquired from different platforms are used as input for the MATLAB-based HRM analysis package. The first set is HRM data of pertactin gene (prn) obtained using a Rotor-Gene 6000 platform, which will henceforth be referred to as the prn data set. There are 21 samples and with a repeat for each one, 42 wells have been used in the assay. Among the 21 samples, there are 11 known prn alleles or variations in the data set [16]. However, only the first 18 wells of the data set which contain 9 of the 11 variants can be identified using the Rotor-Gene software, while prn 5 and 7 in the data set require further processes to identify [16]. The prn alleles and repeat regions are shown in Fig. 2.

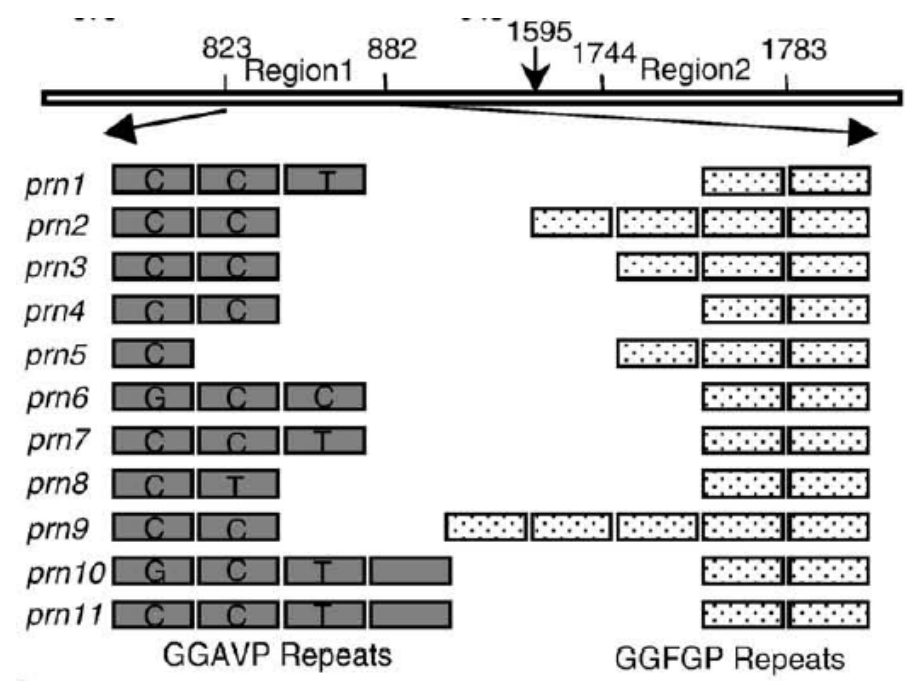

Fig. 2. Schematic of prn alleles and repeat regions contributing to HRM data [16]

The other data set is from a sample data for a web-based application for high resolution data comparison and overlay, uAnalyze [17]. The data was acquired using LightScanner 32-well system and henceforth the data set will be referred to as the LS32 data set. It was noted about the data that the well 29, 30 and 31 contains heterozygous, homozygous and wild type allele respectively. The sequence of the 
allele is given as:

GGAGGAGCTGACCAGTGAAGAAAGTGTCTTTGAAGTCTTCGTTCTTTACC

\section{$\underline{\text { Raw data plot }}$}

As the data files from different sources are in different formats, there is a need for checking validity of the data and identifying the outliers and/or errors. MATLAB function scripts, GetRawData.m, SampleDataGet.m were written to obtain the data from Excel and text file sources. This is facilitated by the MATLAB functions, xlsread( ) and importdata( ) respectively. The temperature and fluorescence data were allocated into separate matrices for other modules, and also for plotting all the raw fluorescence curves with the use of the MATLAB plot( ) function. The current script can be easily changed to accommodate different kinds of data presentation formats. The generated raw data plots are shown in Figs 3 and 4 for the prn data and the LS32 data respectively.

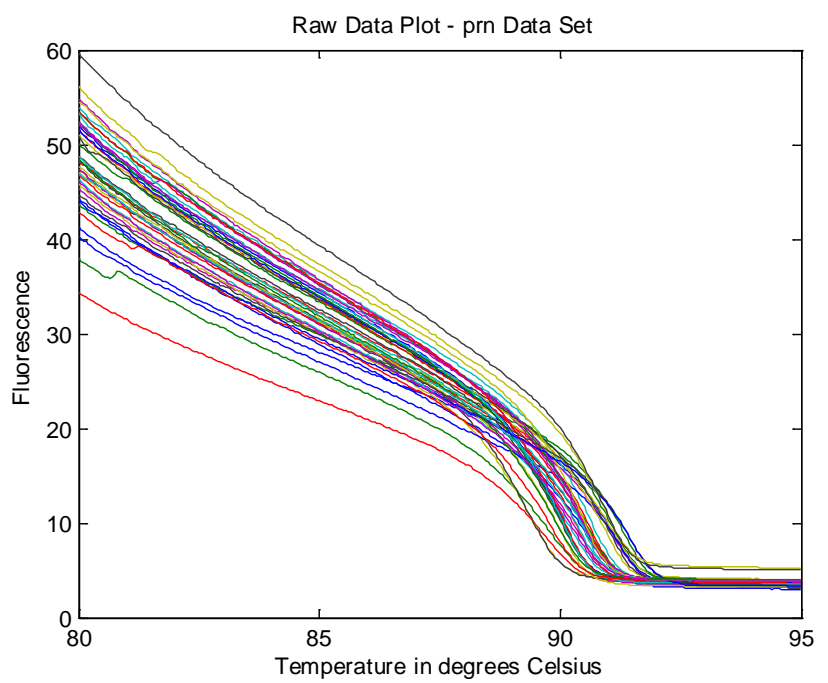

Fig. 3. Raw melt curves of the prn data set 


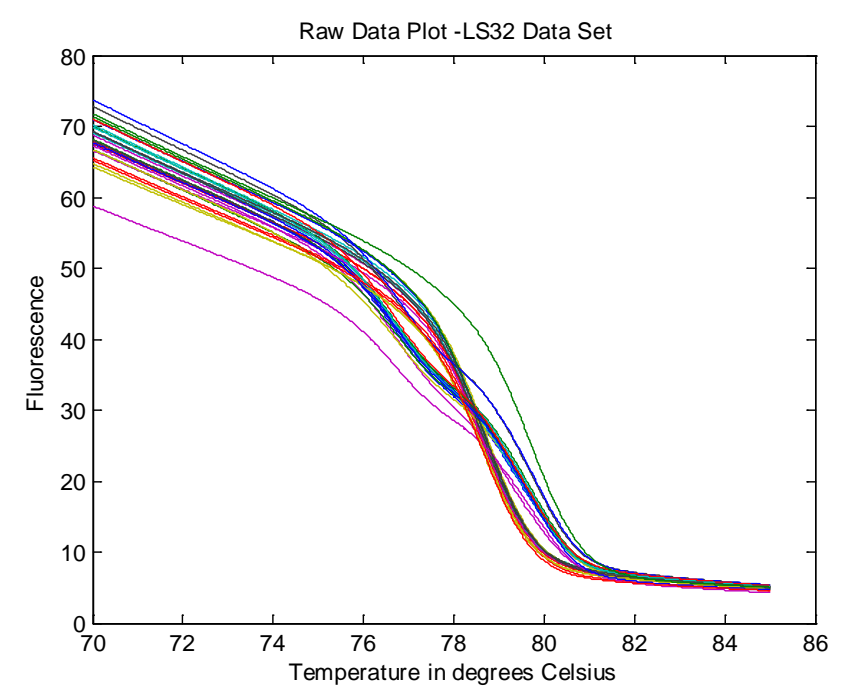

Fig. 4. Raw melt curves of the LS32 data set

There are different assessments in literature in excluding a raw melt curve from analysis. It has been suggested that all samples with fluorescence of less than the $60 \%$ of the maximum should be excluded from the analysis [18]. Plotting the raw melt curve data enables simple and rapid assessment of the general quality of the data and the identification of samples to be excluded from analysis. Depending on the criteria as in [18], a melting curve in red colour shown in Fig. 3 might be excluded as it falls below the $60 \%$ criteria. In the current analysis procedure, the melting curve in question is still used.

\section{$\underline{\text { Melt Region Identification }}$}

Figs 5(a) and 6(a) show the resultant negative first derivative curves of the two data sets. The amplicon melting regions identified by using the developed approach for the well 1 samples are illustrated Figs 7(a) and 8(a), in which the red vertical lines are used to indicate the melting start and end temperatures. In Fig. 7(a), the identified 
melting start and end temperatures for the well 1 sample of the prn data were $89.25{ }^{\circ} \mathrm{C}$ and $92.55{ }^{\circ} \mathrm{C}$ respectively, with a melt region of $3.3{ }^{\circ} \mathrm{C}$. As a comparison, Fig. 7(b) presents the HRM analysis results of the same sample using Rotor-Gene 6000 series software, showing the $\mathrm{dF} / \mathrm{dT}$ curve. It is noted that the normalisation ranges including the leading range and trailing range are defined by users in the Rotor-Gene 6000 software. Both $\mathrm{dF} / \mathrm{dT}$ curves reveal similar trends and close peaks, but it can be seen that there exist clear ripples in the $\mathrm{dF} / \mathrm{dT}$ curve from the Rotor-Gene software. The proposed approach generated much smoother $\mathrm{dF} / \mathrm{dT}$ curve due to the use of the Savitzky-Golay filter. Fig. 8(b) illustrates the dF/dT curve from the HRM analysis result of LS32 well 1 sample using uAnalyze as another comparison, which agrees well with the curve in Fig. 8(a). However, the melting start and end temperatures were not identified by uAnalyze.

(a)

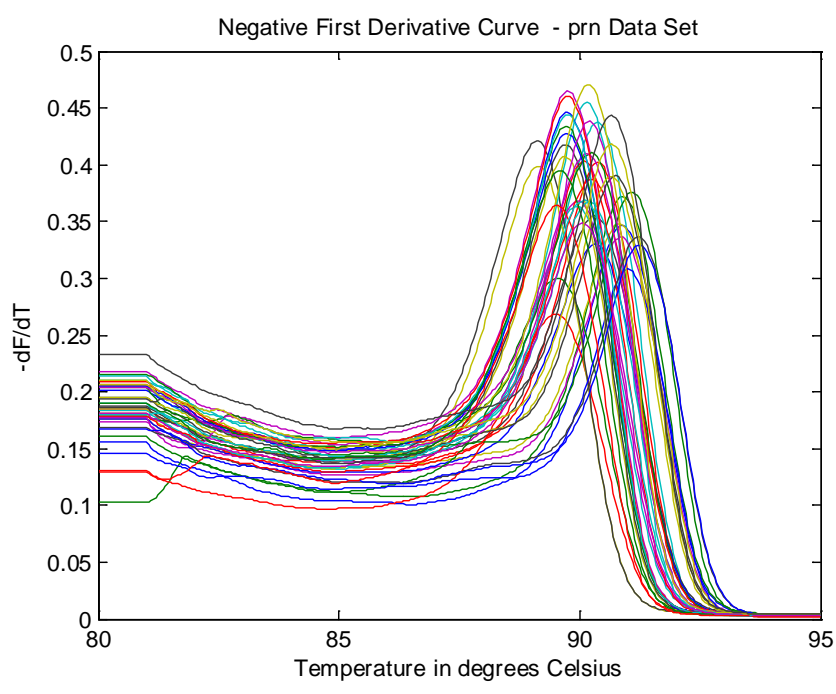




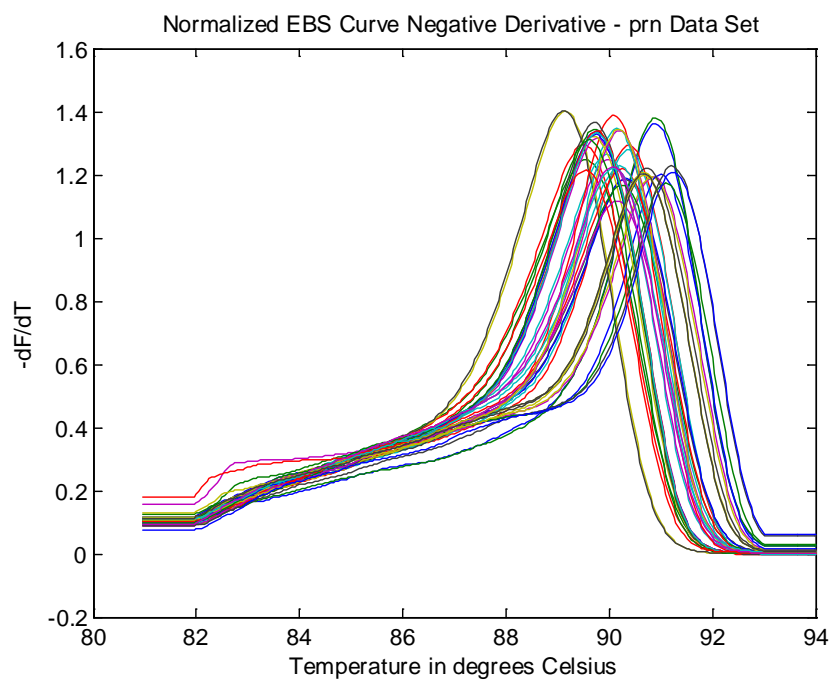

(b)

Fig. 5. (a) Negative first derivative curves of the prn data set; (b) Negative first derivative curves of normalized EBS curves for the prn data set

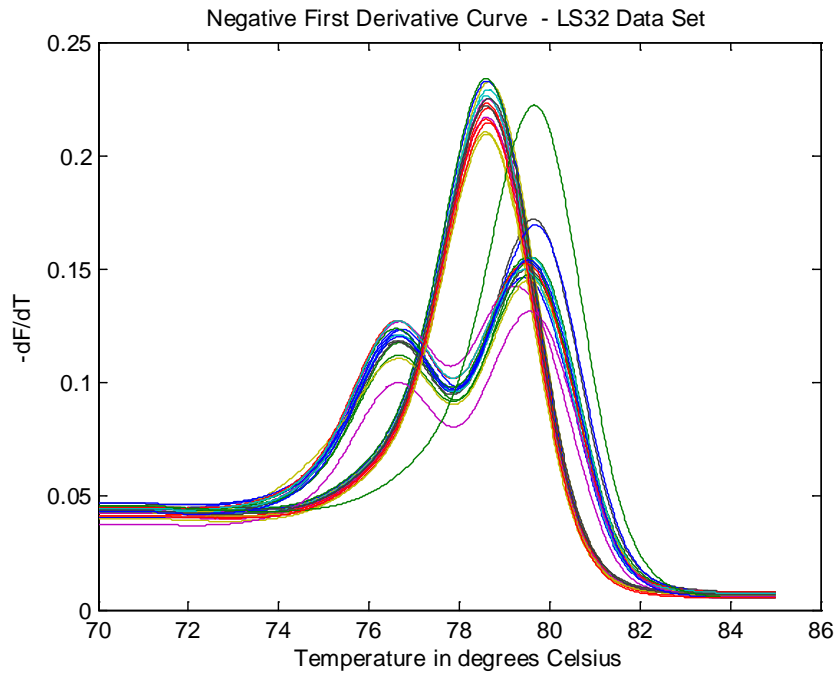

(a) 


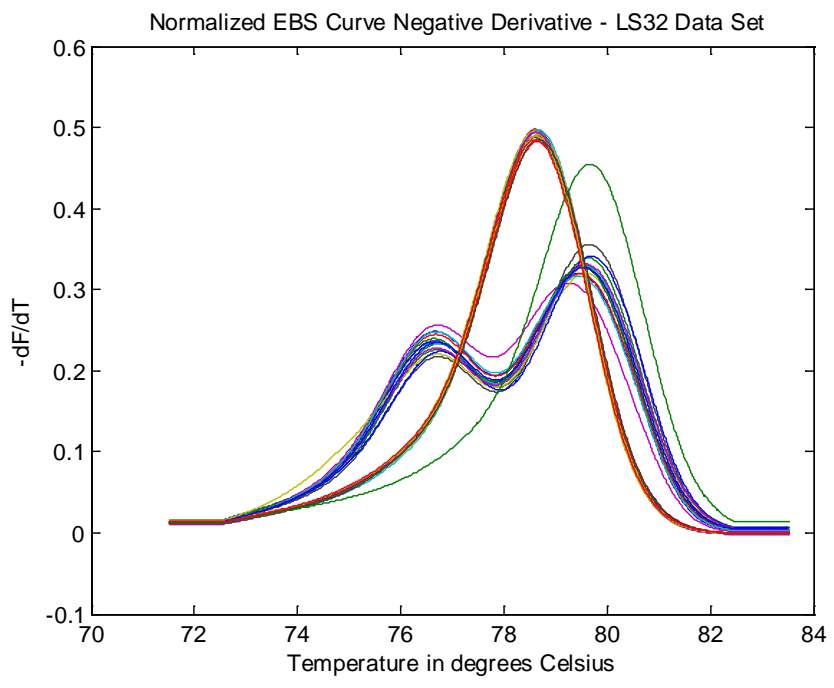

(b)

Fig. 6. (a) Negative first derivative curves of the LS32 data set; (b) Negative first derivative curves of normalized EBS curves for the LightScanner32 data set

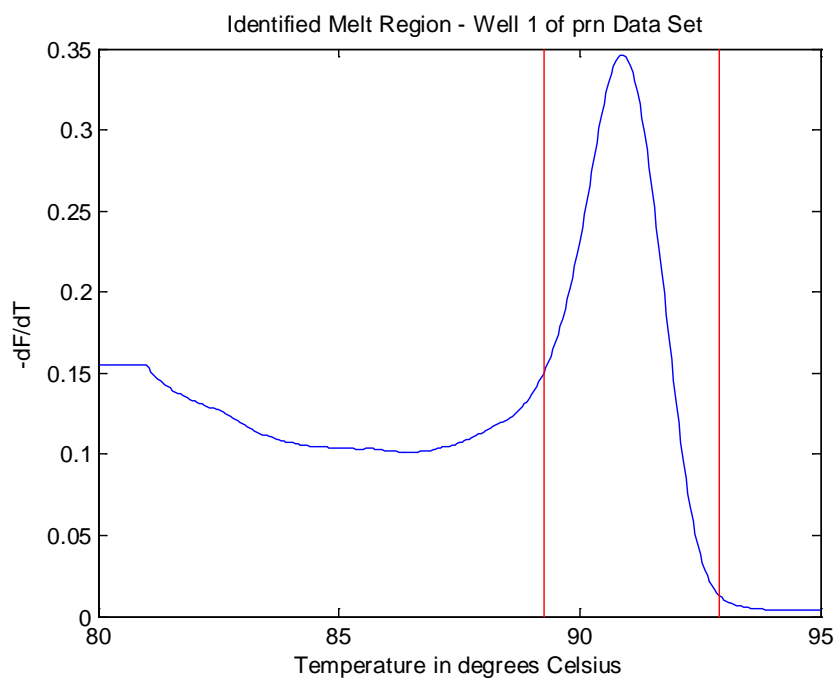

(a) 


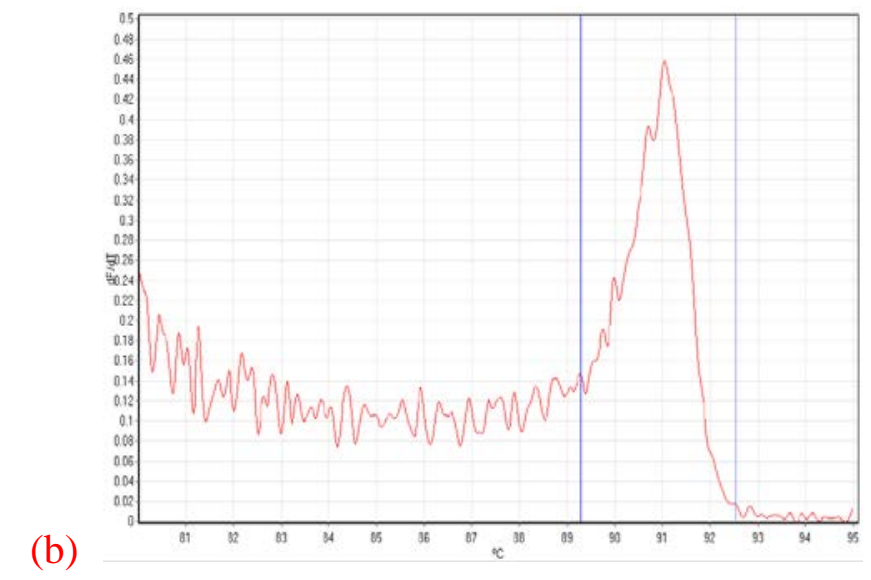

Fig. 7. (a) Identified melting region of well 1 sample in prn data set using the proposed approach; (b) HRM analysis result of well 1 sample using Rotor-Gene 6000 Series Software, showing the $\mathrm{dF} / \mathrm{dT}$ curve. Note that the normalisation ranges including the leading range and trailing range are adjustable by users.

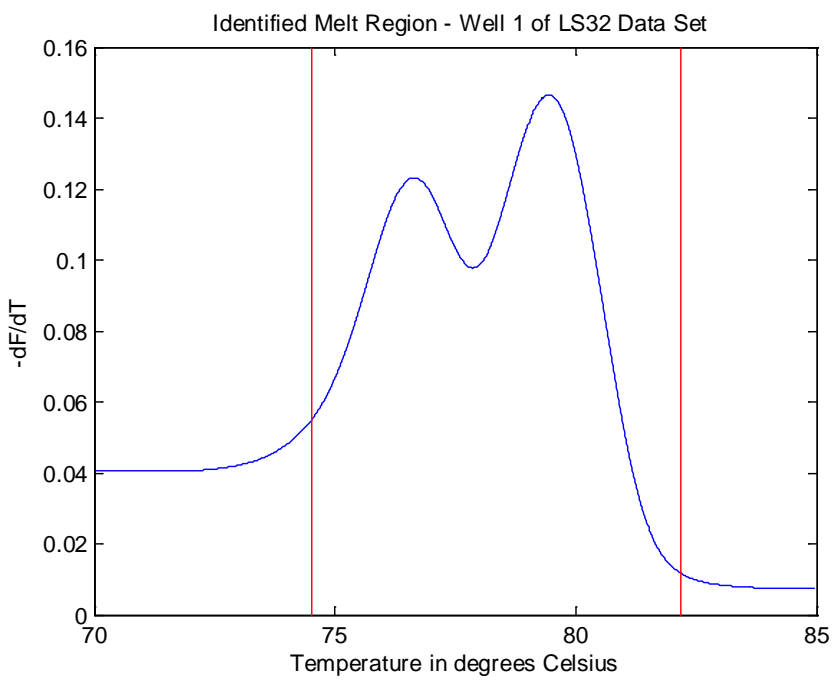

(a) 


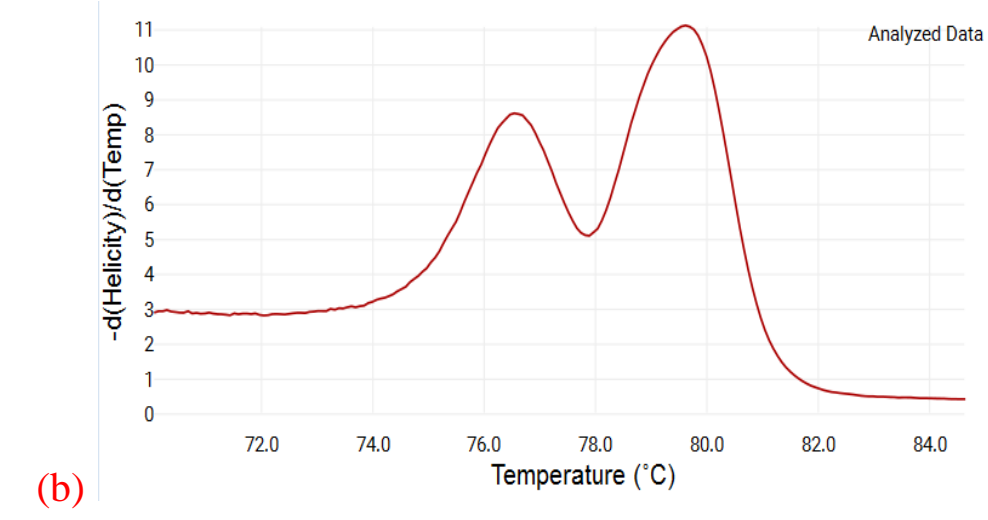

Fig. 8. (a) Identified melting region of well 1 sample in LS32 data set using the proposed approach; (b) HRM analysis result of LS32 well 1 sample using uAnalyze, showing the $\mathrm{dF} / \mathrm{dT}$ curve.

It is evident from the negative first derivative curves that the setup parameters used in the developed algorithm are appropriate, such as the polynomial and window length in the Savitzky-Golay filter, as there is no significant amplified noise in the resultant curves. Some smaller window length parameters have also been assessed which yielded severely amplified noise leading to incorrect results. It is suggested that a window length of $1^{\circ} \mathrm{C}$ temperature range should be suitable in the Savitzky-Golay filter. The identification of the melt regions was successful for all data sets with examples are illustrated in Figs 7 and 8 where the melting region was visually made clear with the red vertical lines. The angles formed by the curves at the identified points were confirmed manually with a protractor.

\section{Exponential Background Subtraction (EBS) and Normalization}


Assessment showed that varying the $T_{L}$ and $T_{R}$ values did not change the exponential background subtracted curves significantly. For the prn data set, a temperature of $80.95^{\circ} \mathrm{C}$ was chosen as $T_{L}$ and $94{ }^{\circ} \mathrm{C}$ as $T_{R}$. For the LS32 data set, $T_{L}$ was set as a temperature of 100 points before the melting start temperature (approximately $71.5^{\circ} \mathrm{C}$ ), and $T_{R}$ was set as 100 points after the melt end at $83.4{ }^{\circ} \mathrm{C}$.

Comparisons of the raw and EBS HRM curves for the first well samples of the prn data set and the LS32 data set are shown in Fig. 9 and Fig. 10 respectively. Furthermore, the normalized raw and normalized EBS curves are also provided. It is clear that the normalized EBS curves are with the superiority of being easier to benchmark. Fig 11(a) displays the final melt curves of the LS32 data after EBS and normalization using the developed program. Normalized plot of LS32 data by using uAnalyze platform is also given in Fig. 11(b). The good agreement of the normalised curves demonstrated the successfulness of the developed approach.

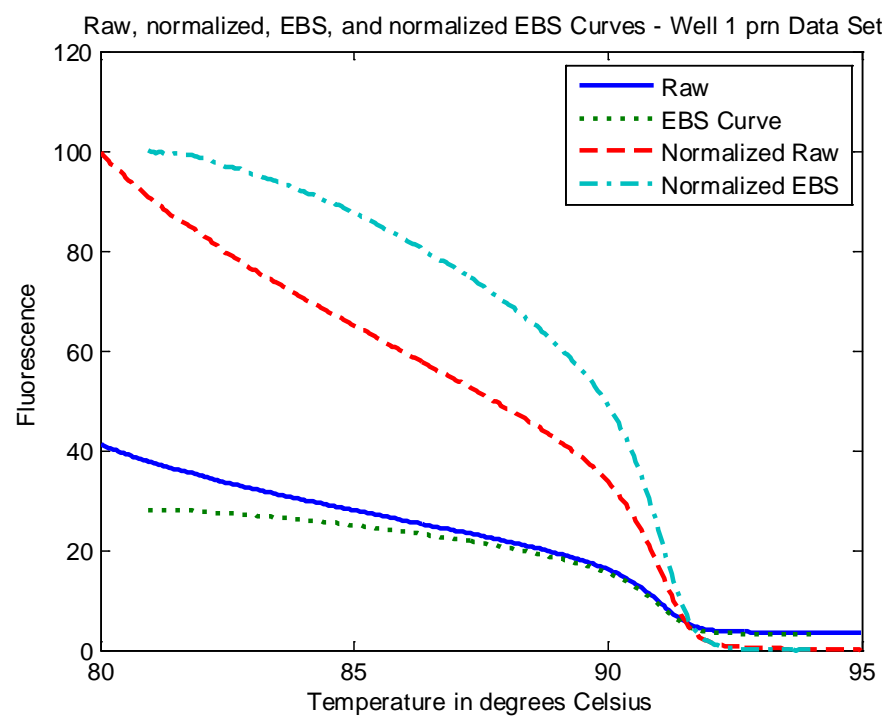

Fig. 9. Raw, EBS, normalized raw and normalized EBS curves for the first well sample of the prn data set 


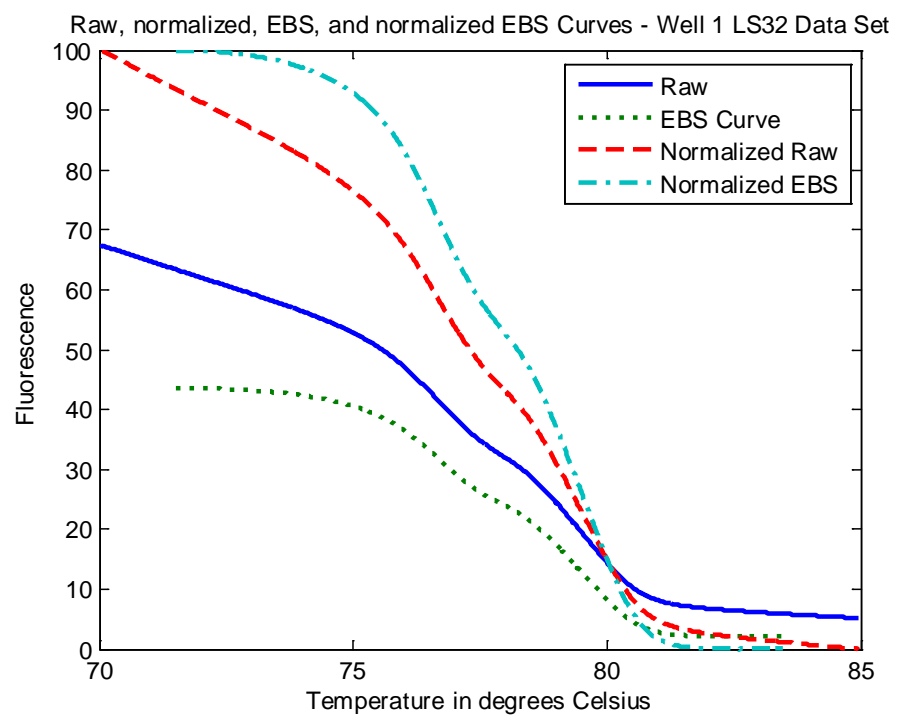

Fig. 10. Raw, EBS, normalized raw and normalized EBS curves for the first well sample of the LS32 data set

It can be observed from the figures that the criterion of proper exponential background removal was fulfilled for all the data sets, which is, horizontal fluorescence before and after duplex melting. Each step of the EBS and normalization was verified by the intermediate resultant plot on the first well data of each data set which displayed the effect of the implemented EBS algorithm and normalization algorithm, as illustrated in Figs. 9 and 10. 
(a)
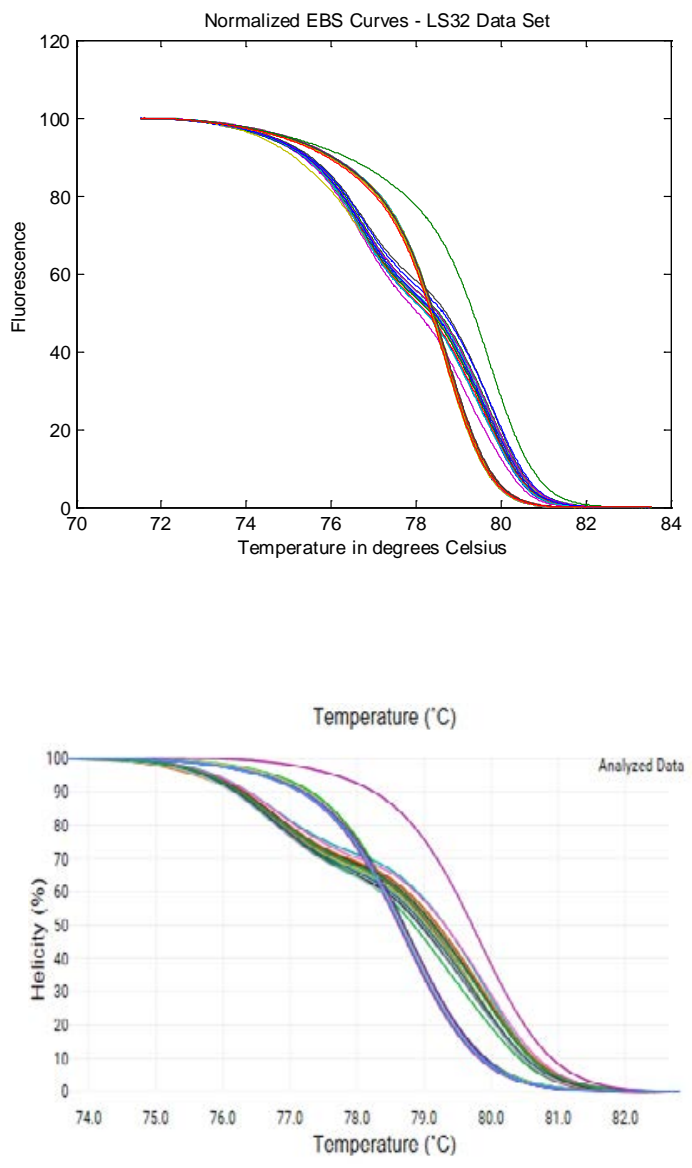

Fig. 11. (a) Normalized exponential background subtracted (EBS) curves of LS32 data set, using the developed program; (b) Normalized plot of LS32 data by using uAnalyze platform

It should be pointed out that the Rotor-Gene 6000 software does not have exponential background subtraction function implemented. The HRM normalised graph shows significant exponential decay behaviour at the start of the melting. In comparison, The HRM normalised graph generated using the developed MATLAB scripts with the EBS demonstrated superiority in this regard. In addition, it is noted that the RotorGene platform and uAnalyze platform could not be used to analyse each other's data sets because of incompatible file formats. This shows the importance of standardisation and an accessible open source code which is the goal of this work. 
The normalized EBS curves allow better visual discrimination based on curve shape in comparison with the raw data. An issue in the current EBS implementation is the step in choosing $T_{L}$ and $T_{R}$ by the user. Although this provides the user with more flexibility, an automatic determination of these temperatures might be more convenient. Also, caution should be taken in the choice as the data is truncated based on the choice of the values.

\section{Melting temperature $\left(T_{m}\right)$ identification}

The negative first derivative curves of normalized EBS curves for the prn and LightScanner32 data are plotted in Figs. 5(b) and 6(b) respectively. Peaks on the derivative curves can be used for the identification of the melting temperatures $\left(T_{m}\right)$. Samples of the identified melting temperatures for all melt curves in the two data sets by using the developed approach are listed in Tables 1 and 2, together with the melting temperatures from the Rotor-Gene 6000 software and uAnalyze for comparison. For simplicity, only the results for the Well 1-12 are shown. Very close agreement of the results can be observed. In the software implementation, melting temperature is determined through the position of curve peaks by using the function $\max ($ ) for the derivative array. It should be noted that there are some issues which might influence the accuracy in the approach. Applying the Savitzky-Golay filter requires ignoring the segments of the melt curve encompassing the Savitzky-Golay window length from the start and end of the data length. Excessive truncation of data with poor choice in user specified temperatures in EBS might cause loss of data up to 
the region of interest. It might result in different values of $T_{m}$ found in different platform.

Table $1 . T_{m}$ of melt curves for prn data set (showing only Well 1-12)

\begin{tabular}{|c|c|c|c|c|}
\hline $\begin{array}{c}\text { Prior Given } \\
\text { Genotype }\end{array}$ & Well & $\begin{array}{c}\text { Tm }\left({ }^{\circ} \mathbf{C}\right) \text { from } \\
\text { proposed } \\
\text { method }\end{array}$ & $\begin{array}{c}\mathbf{T m}^{\prime}\left({ }^{\circ} \mathbf{C}\right) \text { from } \\
\text { Rotor-Gene s/w }\end{array}$ & $\begin{array}{c}\delta \mathrm{Tm}\left({ }^{\circ} \mathbf{C}\right) \\
\left(\mathbf{T m}-\mathbf{T m}^{\prime}\right)\end{array}$ \\
\hline prn 1 & 1 & 90.9 & 91.03 & -0.13 \\
prn 1 & 2 & 90.9 & 91.03 & -0.13 \\
prn 2 & 3 & 90.4 & 90.68 & -0.28 \\
prn 2 & 4 & 90.35 & 90.68 & -0.33 \\
prn 3 & 5 & 90.85 & 91.05 & -0.2 \\
prn 3 & 6 & 90.85 & 91.05 & -0.2 \\
prn 4 & 7 & 90.3 & 90.62 & -0.32 \\
prn 4 & 8 & 90.35 & 90.68 & -0.33 \\
prn 6 & 9 & 89.55 & 89.71 & -0.16 \\
prn 6 & 10 & 89.5 & 89.71 & -0.21 \\
prn 8 & 11 & 89.95 & 90.2 & -0.25 \\
prn 8 & 12 & 89.95 & 90.2 & -0.25 \\
\hline
\end{tabular}

Table 2. $\mathrm{T}_{\mathrm{m}}$ of melt curves for LightScanner data set (showing only Well 1-12)

\begin{tabular}{|c|c|c|c|c|}
\hline $\begin{array}{c}\text { Prior Given } \\
\text { Genotype }\end{array}$ & Well & $\begin{array}{c}\text { Tm }\left({ }^{\circ} \mathrm{C}\right) \text { from } \\
\text { proposed } \\
\text { method }\end{array}$ & $\begin{array}{c}\mathrm{Tm}^{\prime}\left({ }^{\circ} \mathrm{C}\right) \text { from } \\
\text { uAnalyze }\end{array}$ & $\begin{array}{c}\delta \mathrm{Tm}\left({ }^{\circ} \mathrm{C}\right) \\
(\mathrm{Tm}-\mathrm{Tm})\end{array}$ \\
\hline & 1 & 79.47 & 79.65 & -0.18 \\
& 2 & 79.59 & 79.80 & -0.21 \\
& 3 & 78.59 & 78.60 & -0.01 \\
& 4 & 78.63 & 78.60 & 0.03 \\
& 5 & 78.60 & 78.60 & 0.00 \\
& 6 & 78.63 & 78.67 & -0.04 \\
& 7 & 79.62 & 79.72 & -0.10 \\
& 8 & 78.59 & 78.60 & -0.01 \\
& 9 & 78.57 & 78.60 & -0.03 \\
& 10 & 78.63 & 78.60 & 0.03 \\
& 11 & 78.57 & 78.60 & -0.03 \\
& 12 & 79.23 & 79.12 & 0.11 \\
\hline
\end{tabular}

\section{Difference Plots}


(a)
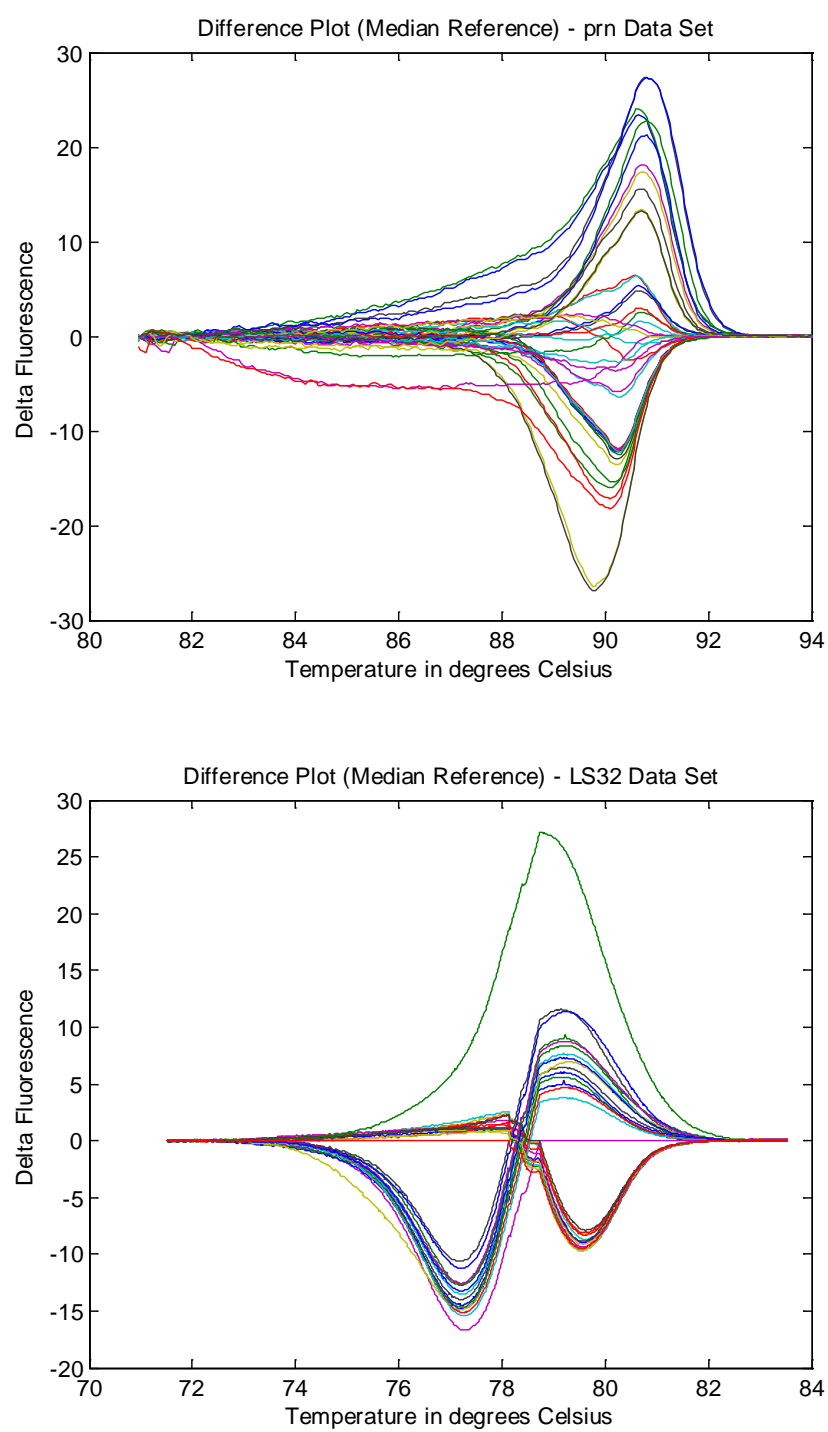

(b)

Fig. 12. Difference plot with composite median reference curve for (a) prn Data Set;

(b) LS32 Data Set

Fluorescence difference plots of the two data sets with the median curve as reference are presented in Fig 12. The difference curves are used to distinguish different genotypes. For the LS32 data set as in Fig. 12(b), there are three clearly different curve clusters that can be easily identified. However, for the prn data set as in Fig. 12(a), there are many variants within the samples, which make the discrimination still a challenge task for the 11 clusters representing all the genotypes. 
The reference curve used for current analysis is the median fluorescence reference curve. It is found that there is no significant difference in visually discriminating genotypes with different reference curves, such as the first well reference curve. In fact, using the median curve can more evenly split the difference curves between positive and negative sides. The reference curve of a known genotype is the choice in most practical analysis cases.

\section{Conclusions}

This paper presents the development of a MATLAB-based open software program for high resolution melting curve analysis. The principle and implementation of key analysis functions, such as to obtain the first derivative curve using Savitzky-Golay filter, to identify the melt region, subtraction of background fluorescence and curve normalization, are introduced. Parameters for Savitzky-Golay filter algorithm to derive first derivative curves are provided and tested. Identification of melt region through angles formed by points of the first derivative curve is successfully performed and validated. The start and end temperatures of the amplicon melting region for each curve can be detected with consistent values.

The current implemented HRM curve analysis program has been assessed by processing HRM data from different platforms. The resultant curves at different stages are presented. In addition, comparison of normalised curves with the results from commercial software is provided to demonstrate the effectiveness of the developed program. The current implementation has the advantages compared to the 
standard commercial software in that it can easily integrate new technological advances and new features to improve the analysis efficiency, reliability, and automation level, which are not in most commercial systems.

\section{Acknowledgement}

The authors would like to thank Mr Vincent Lim for his contribution to this study, and also to thank Dr Sophie Octavia for technical assistance in Rotor-gene HRM analysis.

\section{References}

[1] C.T. Wittwer, G.H. Reed, C.N. Gundry, J.G. Vandersteen, R.J. Pryor, Highresolution genotyping by amplicon melting analysis using LCGreen, Clin. Chem. 49 (2003) 853-860. doi:10.1373/49.6.853.

[2] G.H. Reed, J.O. Kent, C.T. Wittwer, High-resolution DNA melting analysis for simple and efficient molecular diagnostics., Pharmacogenomics. 8 (2007) 597608. doi:10.2217/14622416.8.6.597.

[3] M. Erali, K. V. Voelkerding, C.T. Wittwer, High resolution melting applications for clinical laboratory medicine, Exp. Mol. Pathol. 85 (2008) 5058. doi:10.1016/j.yexmp.2008.03.012.

[4] J.S. Farrar, G.H. Reed, C.T. Wittwer, High-Resolution Melting Curve Analysis for Molecular Diagnostics, Second Edi, Elsevier Ltd, 2003. doi:10.1016/B9780-12-374537-8.00015-8.

[5] Y. Jin, Q. Li, L. Kong, H. Yu, X. Zhong, High-resolution melting (HRM) analysis: A highly sensitive alternative for the identification of commercially important Crassostrea oysters, J. Molluscan Stud. 81 (2015) 167-170. doi:10.1093/mollus/eyu077.

[6] KAPABiosystems, Introduction to High Resolution Melt Analysis, (2013) 17. 
http://kapabiosystems.com/public/pdfs/kapa-hrm-fast-pcr-

kits/Introduction_to_High_Resolution_Melt_Analysis_Guide.pdf.

[7] R. Palais, C.T. Wittwer, Chapter 13 Mathematical Algorithms for HighResolution DNA Melting Analysis, 1st ed., Elesvier Inc., 2009. doi:10.1016/S0076-6879(08)03813-5.

[8] M.M. Cousins, D. Swan, C.A. Magaret, D.R. Hoover, S.H. Eshleman, Analysis of HIV Using a High Resolution Melting (HRM) Diversity Assay: Automation of HRM Data Analysis Enhances the Utility of the Assay for Analysis of HIV Incidence, PLoS One. 7 (2012). doi:10.1371/journal.pone.0051359.

[9] K. De Leeneer, I. Coene, B. Poppe, A. De Paepe, K. Claes, Rapid and sensitive detection of BRCA1/2 mutations in a diagnostic setting: Comparison of two high-resolution melting platforms, Clin. Chem. 54 (2008) 982-989. doi:10.1373/clinchem.2007.098764.

[10] US8068992_Melting curve analysis with exponential background subtraction.pdf, (n.d.).

[11] A. Savitzky, M.J.E. Golay, Smoothing and Differentiation of Data by Simplified Least Squares Procedures., Anal. Chem. 36 (1964) 1627-1639. doi:10.1021/ac60214a047.

[12] D.M. Gray, I. Tinoco, A new approach to the study of sequence-dependent properties of polynucleotides, Biopolymers. 9 (1970) 223-244. doi:10.1002/bip.1970.360090207.

[13] J.H. Mathews, K.D. Fink, Numerical Methods Using MATLAB, (1999) 662.

[14] V. Reja, A. Kwok, G. Stone, L. Yang, A. Missel, C. Menzel, et al., ScreenClust: Advanced statistical software for supervised and unsupervised high resolution $\begin{array}{llllll}\text { melting (HRM) analysis, } & \text { Methods. } 50 \quad \text { (2010) S10-S14. }\end{array}$ doi:10.1016/j.ymeth.2010.02.006.

[15] A.L. Roth, N.D. Hanson, Rapid detection and statistical differentiation of KPC gene variants in gram-negative pathogens by use of high-resolution melting and screenclust analyses, J. Clin. Microbiol. 51 (2013) 61-65. 
doi:10.1128/JCM.02193-12.

[16] W.F. Chan, R.P. Maharjan, P.R. Reeves, V. Sintchenko, G.L. Gilbert, R. Lan, Rapid and accurate typing of Bordetella pertussis targeting genes encoding acellular vaccine antigens using real time PCR and High Resolution Melt $\begin{array}{llllll}\text { analysis, J. } & \text { Microbiol. } & \text { Methods. } & 77 & \text { (2009) }\end{array}$ doi:10.1016/j.mimet.2009.03.007.

[17] uAnalyze Express | Wittwer Lab | Department of Pathology | University of Utah | 2016, (n.d.). https://www.dna.utah.edu/ua/uanalyze.html\# (accessed March 23, 2016).

[18] P.A. Norambuena, J.A. Copeland, P. Krenková, A. Stambergová, M. Macek, Diagnostic method validation: High resolution melting (HRM) of small amplicons genotyping for the most common variants in the MTHFR gene., Clin. Biochem. 42 (2009) 1308-16. doi:10.1016/j.clinbiochem.2009.04.015. 


\section{Figure Captions}

Fig. 1. Flowchart of the HRM data processing procedure

Fig. 2. Schematic of prn alleles and repeat regions contributing to HRM data [16]

Fig. 3. Raw melt curves of the prn data set

Fig. 4. Raw melt curves of the LS32 data set

Fig. 5. (a) Negative first derivative curves of the prn data set; (b) Negative first derivative curves of normalized EBS curves for the prn data set

Fig. 6. (a) Negative first derivative curves of the LS32 data set; (b) Negative first derivative curves of normalized EBS curves for the LightScanner32 data set

Fig. 7. (a) Identified melting region of well 1 sample in prn data set using the proposed approach; (b) HRM analysis result of well 1 sample using Rotor-Gene 6000 Series Software, showing the $\mathrm{dF} / \mathrm{dT}$ curve. Note that the normalisation ranges including the leading range and trailing range are adjustable by users.

Fig. 8. (a) Identified melting region of well 1 sample in LS32 data set using the proposed approach; (b) HRM analysis result of LS32 well 1 sample using uAnalyze, showing the $\mathrm{dF} / \mathrm{dT}$ curve.

Fig. 9. Raw, EBS, normalized raw and normalized EBS curves for the first well sample of the prn data set

Fig. 10. Raw, EBS, normalized raw and normalized EBS curves for the first well sample of the LS32 data set 
Fig. 11. (a) Normalized exponential background subtracted (EBS) curves of LS32 data set, using the developed program; (b) Normalized plot of LS32 data by using uAnalyze platform

Fig. 12. Difference plot with composite median reference curve for (a) prn Data Set; (b) LS32 Data Set 